\title{
Weak Decay Measurements from 2+1 flavor DWF Ensembles
}

\author{
T. Blum ${ }^{1}$, P.A. Boyle ${ }^{2}$, N.H. Christ ${ }^{3}$, J. Frison ${ }^{2}$, N. Garron ${ }^{4}$, T. Izubuchi ${ }^{5,6}$, \\ T. Janowski ${ }^{7}$, A. Juettner ${ }^{7}$, C. Jung ${ }^{6}$, C. Kelly ${ }^{3}$, C. Lehner ${ }^{5,6}$, R.D. Mawhinney ${ }^{* 3}$, \\ G. McGlynn ${ }^{3}$, D. Murphy ${ }^{3}$, S. Ohta ${ }^{8,9,5}$ C.T. Sachrajda ${ }^{7}$, A. Soni ${ }^{6}$, H. Yin ${ }^{3}$ \\ ${ }^{1}$ Physics Department, University of Connecticut, Storrs, CT 06269-3046, USA \\ ${ }^{2}$ SUPA, School of Physics, The University of Edinburgh, Edinburgh EH9 3JZ, UK \\ ${ }^{3}$ Physics Department, Columbia University, New York, NY 10027, USA \\ ${ }^{4}$ School of Mathematics, Trinity College, Dublin, Ireland \\ ${ }^{5}$ RIKEN-BNL Research Center, Brookhaven National Laboratory, Upton, NY 11973, USA \\ ${ }^{6}$ Brookhaven National Laboratory, Upton, NY 11973, USA \\ ${ }^{7}$ School of Physics and Astronomy, University of Southampton, Southampton SO17 1BJ, UK \\ ${ }^{8}$ Institute of Particle and Nuclear Studies, KEK, Tsukuba, Ibaraki, 305-0801, Japan \\ ${ }^{9}$ Sokendai Graduate University of Advanced Studies, Hayama, Kanagawa 240-0193, Japan
}

The RBC and UKQCD Collaborations, ${ }^{\dagger}$

\begin{abstract}
Since March of 2012, the RBC and UKQCD collaborations have produced two 2+1 flavor Mobius domain wall fermion ensembles using BGQ computers at the University of Edinburgh, Brookhaven National Laboratory, the RIKEN-BNL Research Center and Argonne National Laboratory. These are $2+1$ flavor ensembles at essentially the physical pion and kaon masses and have volumes of $(5.5 \mathrm{fm})^{3}$. The evolutions currently contain more than 1,300 thermalized molecular dynamics time units. An extensive set of observables, $m_{\pi}, m_{K}, m_{\Omega}, f_{\pi}, f_{K}, B_{K}, f_{+}^{K \pi}(0)$ (the $K_{l 3}$ form factor at $q^{2}=0$ ) and the $\Delta I=3 / 2, K \rightarrow \pi \pi$ real and imaginary amplitudes are being calculated, all in a common measurement package that uses EigCG and all mode averaging to speed up these calculations by a factor of $5-10 \times$ over our previous methods. This note will describe our ensembles, our measurement package and give preliminary results from this calculation.
\end{abstract}

31st International Symposium on Lattice Field Theory - LATTICE 2013

July 29 - August 3, 2013

Mainz, Germany

\footnotetext{
* Speaker.

${ }^{\dagger}$ We gratefully acknowledge computer support in the US from the ALCF at ANL, BNL and the RIKEN-BNL Research Center and in the UK through the STFC funded DiRAC facility. Individual researchers received support from the US DOE, UK STFC and the EU.
} 


\section{Introduction}

The RBC and UKQCD Collaborations have been generating a series of 2+1 flavor QCD ensembles with domain wall fermions and the Iwasaki gauge action since 2005. The first two series of these ensembles, with $a^{-1}=1.7 \mathrm{GeV}$ and $2.3 \mathrm{GeV}$, used dynamical pion masses as light as $330 \mathrm{MeV}$ and $290 \mathrm{MeV}$, respectively [1]. By adding a dislocation suppressing determinant ratio (DSDR) term to the action, we were able to simulate on a coarser lattice $\left(a^{-1}=1.3 \mathrm{GeV}\right)$, with dynamical pions as light as $170 \mathrm{MeV}$ [2]. By combining this DSDR ensemble with our two previous ensembles, we were able to markedly reduce our chiral extrapolation errors. We performed global fits to $m_{\pi}, m_{K}, m_{\Omega}, f_{\pi}$ and $f_{K}$ on these three ensembles, expanding about the physical, continuum theory using $\mathrm{SU}(2)$ chiral perturbation theory (ChPT) and also keeping $O\left(a^{2}\right)$ corrections. (Our DSDR ensemble, since it has a different action, has different values for the $O\left(a^{2}\right)$ coefficients than our other two ensembles.) We used $m_{\pi}, m_{K}$ and $m_{\Omega}$ to set the scale and the (degenerate) light quark mass and strange quark mass. In addition to finding $f_{\pi}=132.1(3.8) \mathrm{MeV}, f_{K}=152.4(3.4) \mathrm{MeV}$, determining the quark masses and some of the low energy constants (LECs) of SU(2) ChPT, we were also able to determine $\operatorname{Re}\left(A_{2}\right)$ and $\operatorname{Im}\left(A_{2}\right)$ for $K \rightarrow \pi \pi$ decays [3]. In addition $f_{+}^{K \pi}(0)$ was measured and found to be $0.9679(20)_{\text {stat }}\left({ }_{-46}^{+18}\right)_{\text {stat }}$ [4].

To further reduce the errors on these quantities, we have been producing $2+1$ flavor ensembles at essentially the physical quark masses, with $a^{-1}=1.7$ and $2.3 \mathrm{GeV}$. To keep the contribution of the residual mass, $m_{\text {res }}$, to the light quark mass at the $\sim 30 \%$ level, we have been generating our new ensembles with Mobius domain wall fermions (MDWF) [5] and the Iwasaki gauge action. To make measurements on these two essentially physical quark mass ensembles, we have developed a measurement package that uses EigCG to produce low modes for deflation for the light quarks. We then use the all mode averaging (AMA) method of [6] to optimize the contribution to the error between high precision light-quark propagator solves and reduced precision solves. This has allowed us to make measurements $5-10 \times$ faster than our previous methods. This has been a vital improvement, since the statistical errors for many of our observables have increased markedly as the light quark mass has been reduced.

In this report, we first describe our ensemble generation, followed by our measurement methods. We then use the global fit strategy we have described in $[1,2]$, to do the small extrapolation from the parameters for these new ensembles to the continuum and chiral limits.

\section{Ensembles}

In Table 1, we list the 2+1 flavor Iwasaki gauge action ensembles that the RBC and UKQCD Collaborations have produced and are producing, along with a stronger coupling ensemble (13) that is being produced by the RBC and HotQCD Collaborations. Here we will focus on ensembles 10 and 11, generated with MDWF using values of $b=1.5$ and $c=0.5$ for the Mobius parameters, so that $b-c=1$. As shown in [7], with these parameters the MDWF and DWF approximations to the sign function differ fractionally by less than $10^{-3}$ over the entire range of eigenvalues of the kernel $\gamma_{5} D_{W} /\left(2+D_{W}\right)$ Thus our ensemble 10 with MDWF and $L_{s}=24$ very accurately represents a DWF ensemble with $L_{s}=48$ and ensemble 11 corresponds to an $L_{s}=24$ DWF ensemble. The residual 


\begin{tabular}{|c|c|c|c|c|c|c|c|c|}
\hline \multirow[t]{2}{*}{ Ens. } & \multirow{2}{*}{$\begin{array}{l}\text { Action } \\
(\mathrm{G}+\mathrm{F})\end{array}$} & \multirow{2}{*}{$\begin{array}{c}1 / a \\
(\mathrm{GeV})\end{array}$} & \multirow{2}{*}{$\begin{array}{l}\text { Lattice } \\
\text { volume }\end{array}$} & $m_{l}$ & $m_{s}$ & $m_{\text {res }}$ & \multirow{2}{*}{$\begin{array}{c}m_{\pi} \\
(\mathrm{MeV})\end{array}$} & \multirow{2}{*}{$\begin{array}{l}\text { Size } \\
(\mathrm{fm})\end{array}$} \\
\hline & & & & \multicolumn{3}{|c|}{ (in lattice units) } & & \\
\hline 1 & DWF+I & $1.75(3)$ & $24^{3} \times 64 \times 16$ & 0.005 & 0.04 & 0.00308 & 330 & 2.7 \\
\hline 2 & $\mathrm{DWF}+\mathrm{I}$ & $1.75(3)$ & $24^{3} \times 64 \times 16$ & 0.01 & 0.04 & 0.00308 & 420 & 2.7 \\
\hline 3 & $\mathrm{DWF}+\mathrm{I}$ & $1.75(3)$ & $24^{3} \times 64 \times 16$ & 0.02 & 0.04 & 0.00308 & 560 & 2.7 \\
\hline 4 & DWF+I & $1.75(3)$ & $24^{3} \times 64 \times 16$ & 0.03 & 0.04 & 0.00308 & 670 & 2.7 \\
\hline 5 & $\mathrm{DWF}+\mathrm{I}$ & $2.31(4)$ & $32^{3} \times 64 \times 16$ & 0.004 & 0.03 & 0.000664 & 310 & 2.6 \\
\hline 6 & DWF+I & $2.31(4)$ & $32^{3} \times 64 \times 16$ & 0.006 & 0.03 & 0.000664 & 370 & 2.6 \\
\hline 7 & DWF+I & $2.31(4)$ & $32^{3} \times 64 \times 16$ & 0.008 & 0.03 & 0.000664 & 420 & 2.6 \\
\hline 8 & DWF+ID & $1.37(1)$ & $32^{3} \times 64 \times 32$ & 0.0042 & 0.046 & 0.00184 & 250 & 4.5 \\
\hline 9 & DWF+ID & $1.37(1)$ & $32^{3} \times 64 \times 32$ & 0.001 & 0.046 & 0.00184 & 180 & 4.5 \\
\hline 10 & MDWF+I & $1.75(3)$ & $48^{3} \times 96 \times 24$ & 0.00078 & 0.0362 & 0.000614 & 138 & 5.5 \\
\hline 11 & MDWF+I & $2.31(4)$ & $64^{3} \times 128 \times 12$ & 0.000678 & 0.02661 & 0.000314 & 139 & 5.5 \\
\hline 12 & DWF+I & $3.06(6)$ & $32^{3} \times 64 \times 12$ & 0.0047 & 0.0186 & 0.00060 & 380 & 2.0 \\
\hline 13 & MDWF+ID & $1.12(4)$ & $32^{3} \times 64 \times 24$ & 0.00022 & 0.05960 & 0.0021 & 135 & 5.8 \\
\hline
\end{tabular}

Table 1: Dynamical 2+1 flavor domain wall fermion ensembles produced (1-9) and being produced (10-13) by the RBC and UKQCD collaborations (10-12) and the RBC and HotQCD collaborations (13). The gauge and fermion $(\mathrm{G}+\mathrm{F})$ action abbreviations are: $\mathrm{DWF}=$ domain wall fermions, $\mathrm{MDWF}=$ Mobius domain wall fermions, I = Iwasaki gauge action, ID = Iwasaki plus Dislocation Suppressing Determinant Ratio (DSDR) gauge action. The total light quark mass (in lattice units) is $m_{l}+m_{\text {res }}$ and the total strange quark mass is similarly $m_{s}+m_{\text {res }}$.

mass, $m_{\text {res }}$, for ensemble 10 is about $45 \%$ of the physical light quark mass, while for ensemble 11 $m_{\text {res }}$ is about $30 \%$ of the physical light quark mass.

We now give some of the details for the generation of ensembles 10 and 11 . We use an exact hybrid Monte Carlo algorithm for our ensemble generation, with 5 intermediate Hasenbusch masses $(0.005,0.017,0.07,0.18,0.45)$ for the 2 flavor part of the algorithm for both ensembles and a rational approximation for the strange quark. For ensemble 10, each trajectory requires a total of $5.9 \times 10^{5} \mathrm{CG}$ iterations, while for ensemble $11,6.1 \times 10^{5}$ iterations are required. For ensemble 10 , we use 15 steps per trajectory, with $\Delta \tau=0.067$, giving an acceptance of $84 \%$, while for ensemble 11, each trajectory is 9 steps with $\Delta \tau=0.111$ yielding an $87 \%$ acceptance. We have 1,300 thermalized trajectories for ensemble 10 and 1,400 for ensemble 11. A trajectory takes 3.5 hours on 2 racks of BGQ for ensemble 10 and 0.67 hours on 8 racks for ensemble 11 .

To check the ergodicity of the HMC/RHMC algorithm for these light quarks, Figure 1 shows the evolution of the global topological charge for trajectories 1,200 to 2,200 for ensemble 11. (The first 1,100 trajectories of ensemble 11 were generated with light quarks that gave a $170 \mathrm{MeV}$ pion mass.) This plot suggests that $O(800)$ molecular dynamics time units are required to do one sample of the range of topologies possible for these parameters. In Figure 2, we plot an estimate of the topological susceptibility for ensemble 11 (filled point) along with earlier results at heavier quark masses, including LO and NLO ChPT fits to the massless limit. While we have not attempted to place an error bar on the ensemble 11 point, it is encouraging that it is close to the expected value. 


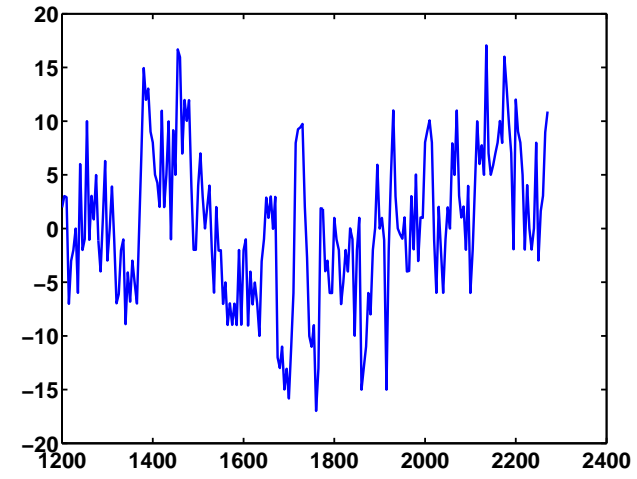

Figure 1: Topological charge evolution on ensemble 11 .

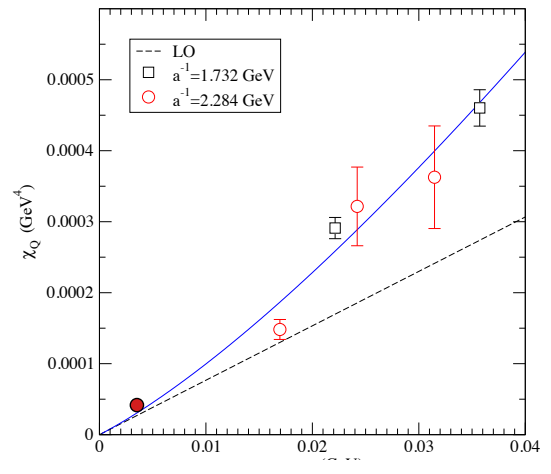

Figure 2: Chiral susceptibility for ensemble 11 (filled point), along with results from [1] for larger masses and fits to LO and NLO ChPT.

\section{Measurement Package}

The ability to generate ensemble 10 and 11 forced us to seek dramatic improvements in our measurement strategy, since the statistical error for kaon observables increases with decreasing light quark mass (holding the strange quark mass fixed). By an analogous argument to the one used for large errors for nucleon observables, for zero momentum kaons the error depends on one half the mass of the pion plus $\bar{s} s$ state compared to the kaon state. This effect is larger for kaons made from quarks with momentum, which are required for measurements of $K \rightarrow \pi \pi$ decays and $f_{+}^{K \pi}(0)$, since the noise is controlled by zero momentum pion states compared to the heavier nonzero momentum kaon states.

The first part of our measurement package strategy involves maximally reusing propagators for all of the measurements we are doing, which includes $m_{\pi}, m_{K}, m_{\Omega}, f_{\pi}, f_{K}, B_{K}, f_{+}^{K \pi}(0)$ and $K \rightarrow$ $(\pi \pi)_{I=2}$. Reusing propagators requires choosing a common source for these observables which is satisfactory for all of them. Also, since we will be measuring both 2 and 3 point functions, we need to be able to control the spatial momentum of the sources to project out unwanted momenta. We did numerous studies of Coulomb gauge fixed wall sources and Coulomb gauge fixed box sources for many of these observables. (The box sources were generically chosen so that an integer multiple of their linear dimension would fit in the lattice volume, allowing us to get zero momentum projections by using all possible box sources.) While the box sources showed faster projection onto the desired ground state, the statistical errors on the wall sources were much smaller, so that the achieved error on the measured quantity per unit of computer time was essentially the same. From these studies, we chose to use the simple Coulomb gauge fixed wall sources.

In previous work on the $\eta-\eta^{\prime}$ mass, which involves disconnected quark diagrams, we had found that translating $n$ point functions over all possible temporal source locations reduced the error essentially as the square root of the number of translations, for this noisy result [8]. The calculation of this many quark propagators on a single lattice can be accomplished much more quickly by a deflation algorithm. The EigCG algorithm [9] was used for $K \rightarrow(\pi \pi)_{I=0,2}$ measurements at unphysical kinematics in [10]. Measurements were again done for all temporal translations of 
the $n$ point functions and a factor of 7 speed-up was measured. Here, the EigCG algorithm was chosen rather than the inexact deflation of [11] for two reasons: the inexact deflation scheme has not (yet) been optimally formulated for the DWF operator and even given such a formulation, an efficient parallel implementation could be challenging. By contrast, EigCG is straightforward to parallelize, with the major drawback being the large memory footprint it requires. However, large BGQ partitions have large memory so this issue can be managed.

Thus we were led to an approach where we would calculate all temporal translations of all $n$ point functions on a single configuration, using EigCG to deflate the solves. We are not doing measurements with disconnected diagrams here, but we do have non-zero momentum in some of our propagators, and we found that temporal translations gave substantial reductions in statistical errors, depending on the observable. The final ingredient in our measurement package is the inclusion of the AMA techniques of [6]. We employ these ideas to calculate most of our $n$ point functions using a loose or sloppy CG solve, where the residual is $10^{-4}$ or $10^{-5}$. These sloppy solves are done after deflation, so they are somewhat more accurate than the residual indicates, since many low modes have already been projected out. We calculate a few exact solves, with a residual of $10^{-8}$, to correct for the error from the sloppy solves. One optimizes the errors and costs between the sloppy and exact solves. The final result for the $n$ point function is an exact result - the optimization is only on the size of the error. To avoid any biases due to the even-odd decomposed Dirac operator that is actually used in the CG we do two things. The first is that we use EigCG on a volume source to calculate the eigenvectors for deflation. The second is that the temporal slices where we do the exact solves are chosen randomly for each lattice.

We are left with the following strategy. Our calculation requires light quarks with 3 different momenta: $p=0, p_{K l 3}$ (the momentum for the light quark in $\left\langle K\left|s \gamma_{\mu} d\right| \pi\right\rangle$ to have $q^{2}=0$ at the vertex), and $p_{K \pi \pi}$ (the physical momentum carried by the pions in this decay for $I=2$ ). For each light quark momentum we 1) use EigCG on a volume source to produce the desired number of low modes and 2) do exact, deflated light quark solves for a few temporal locations of a Coulomb gauge fixed wall source and 3) do all possible sloppy light quark solves for Coulomb gauge fixed wall sources. We calculate low modes in single precision using EigCG (to reduce the memory footprint) and also do sloppy solves in single precision. For exact solves, we achieve double precision accuracy through multiple restarts of single precision solves, restarting the solve by correcting the defect as calculated in double precision. For the zero-momentum strange quark propagators required, we do a standard, accurate CG solve for sources on every time slice. On ensemble 10, we have done measurements using single rack BGQ partitions (1024 nodes), calculating 600 low modes with EigCG (which fills the memory) and running continuously for 5.5 days. For ensemble 11, measurements have been done on between 8 and 32 rack BGQ partitions at the ALCF and 1500 low modes are calculated by EigCG. On a 32 rack partition, the measurements take 5.3 hours and the solver sustains 1 PFlops.

\section{Results for Basic Measurements}

For our input simulation parameters for ensembles 10 and 11 ( $\beta=2.13$ and 2.25, respectively), we used the results for the physical quark masses for these couplings from the global fits in [2]. The output results for the ratios of $m_{\pi}, m_{K}$ and $m_{\Omega}$ from ensembles 10 and 11 are compared with 
physical values in Table 2. Clearly we are very close to the physical ratios, but we require a method to do this very modest extrapolation to the precise physical ratios. Since we are not including electromagnetic effects in our fits, we use the following physical values as inputs: $m_{\pi}=135.0$ $\mathrm{MeV}, m_{K}=495.7 \mathrm{MeV}$ and $m_{\Omega}=1.67225 \mathrm{GeV}$.

In $[1,2]$, we have extensively described the global fits to our heavier quark mass data, using SU(2) NLO chiral perturbation theory and $O\left(a^{2}\right)$ corrections to the continuum. Here, we use a simple variant of these fits to get preliminary results for quantities at physical quark masses, including the new results from ensembles 10 and 11 . We use the same fit functions and all data from ensembles 1 to 11 with $m_{\pi}<350 \mathrm{MeV}$, but for the fit to each individual jackknife block we strongly overweight the ensemble 10 and 11 data. This forces the fit to accurately go through the ensemble 10 and 11 data points jackknife block by jackknife block, while effectively increasing the error on the heavier mass points. This is a sensible approach, since NLO ChPT is not arbitrarily accurate for pions with masses in the $200-350 \mathrm{MeV}$ range, but rather is expected to be off by $O(5 \%)$. Given that we are only extrapolating a few percent in quark mass from the ensemble 10 and 11 results any inaccuracies in the LEC's from NLO should be very small in the final result. With this overweighted fit, the statistical error on our results is dominantly the statistical error of ensemble 10 and 11 . We will explore this and other fitting strategies more thoroughly after we have our final data.

Employing this fit strategy and including measurements on 51 configurations of ensemble 10 , separated by $20 \mathrm{MD}$ time units, and 28 configurations of ensemble 11, separated by $40 \mathrm{MD}$ time units we find the following values in the continuum limit at the physical point: $f_{\pi}=130.3 \pm 1.7$ $\mathrm{MeV}, f_{K}=155.7 \pm 1.8 \mathrm{MeV}$ and $f_{K} / f_{\pi}=1.195 \pm 0.005$. The errors here are statistical only and the fits include data from [2] for quark masses such that $m_{\pi}<350 \mathrm{MeV}$. We can also compare the statistical error from the few exact calculations we do on each lattice to the total result, including all the sloppy solves. For $m_{\pi}, m_{K}, f_{\pi}$, and $f_{K}, \sigma_{\text {exact }} / \sigma_{\text {total }} \sim 2-3$, for $B_{K}$ this ratio is 10 , and for $f_{+}^{K \pi}(0)$ it is around 5. Compared to previous measurements and including the additional cost of the EigCG setup, this approach is giving us a speed-up of $5-10 \times$ for many of our observables.

\begin{tabular}{ccccccc}
\hline \multirow{2}{*}{ Quantity } & Physical & \multicolumn{2}{c}{$48^{3}$} & & \multicolumn{2}{c}{$64^{3}$} \\
\cline { 3 - 4 } \cline { 6 - 7 } & value & ratio & deviation & ratio & deviation \\
\hline$m_{\pi} / m_{K}$ & 0.2723 & $0.2797(7)$ & $2.7 \%$ & & $0.2739(12)$ & $0.6 \%$ \\
$m_{\pi} / m_{K}$ & 0.0807 & $0.0828(5)$ & $2.5 \%$ & & $0.0821(5)$ & $1.7 \%$ \\
$m_{K} / m_{\Omega}$ & 0.2964 & $0.2959(18)$ & $-0.2 \%$ & & $0.2997(14)$ & $1.1 \%$
\end{tabular}

Table 2: Ratios of $m_{\pi}, m_{K}$ and $m_{\Omega}$ from our simulations and the physical values.

\section{Results for Weak Decays}

Here we summarize in Table 3 the status for other kaon observables that have been calculated as part of our measurement package. More details on each of these are reported in other contributions to this conference, referenced below.

For $B_{K}(\overline{\mathrm{MS}}, 3 \mathrm{GeV})$ [12], we only require zero momentum light quarks and find the measurement strategy outlined here has reduced the statistical errors, per configuration, by a factor of 10 . 
This shows up as a reduction of 2.7 in the final statistical error on $B_{K}$. We also have a number of temporal separations between the $K^{0}$ and $\bar{K}^{0}$ source to allow better understanding of systematic errors.

For $f_{+}^{K \pi}(0)$, we have used non-zero momentum on one light quark in the pion, via twisted boundary conditions, to achieve the desired value $q^{2}=0$. The ensemble 10 and 11 points, as discussed in [13], agree very well with the central value previously quoted and should provided a noticeable reduction in the previous systematic errors, which largely come from the chiral extrapolation. Preliminary results for $K \rightarrow \pi \pi_{(I=2)}$ using ensembles 10 and 11 are reported in [14]. Here antiperiodic boundary conditions in all three spatial directions are needed to achieve the required physical momentum for the pions in the final state. Although the analysis is in a preliminary stage, the central values agree well with previous results and the new data allows a continuum limit to be taken, so the large $O\left(a^{2}\right)$ errors in the previous result will be markedly reduced.

\begin{tabular}{|c|c|c|}
\hline Quantity & Previous Result & $\begin{array}{l}\text { Preliminary Result } \\
\text { (including ensembles } 10 \text { and 11) }\end{array}$ \\
\hline$B_{K}$ & $0.535(8)_{\text {stat }}(7)_{\chi}(3)_{\mathrm{FV}}(11)_{\text {pert }}$ & $0.533(3)_{\text {stat }}(0)_{\chi}(3)_{\mathrm{FV}}(11)_{\text {pert }}$ \\
\hline$f_{+}^{K \pi}(0)$ & $0.9670(20)_{\text {stat }}\left({ }_{-42}^{+0}\right)_{\text {model }}(7)_{\mathrm{FSE}}(17)_{\text {cutoff }}$ & Reduction in model errors \\
\hline $\operatorname{Re} A_{2}$ & $1.381(46)_{\mathrm{stat}}(135)_{\mathrm{sys}}(207)_{a^{2}} \times 10^{-8} \mathrm{GeV}$ & Comparable central value, no $a^{2}$ error. \\
\hline $\operatorname{Im} A_{2}$ & $-6.54(46)_{\mathrm{stat}}(72)_{\mathrm{sys}}(98)_{a^{2}} \times 10^{-13} \mathrm{GeV}$ & Comparable central value, no $a^{2}$ error. \\
\hline
\end{tabular}

Table 3: A comparison of previous and new preliminary results for some kaon observables.

\section{References}

[1] Y. Aoki, et. al., RBC-UKQCD Collaboration, Phys. Rev. D83 (2011) 074508.

[2] R. Arthur, et. al., RBC-UKQCD Collaboration, Phys. Rev. D87 (2013) 094514.

[3] T. Blum, et. al., RBC-UKQCD Collaboration, Phys. Rev. Lett. 108 (2012) 141601, T. Blum, et. al., RBC-UKQCD Collaboration, Phys. Rev. D86 (2012) 074513.

[4] P.A. Boyle, et. al., RBC-UKQCD Collaboration, JHEP 08 (2013) 132.

[5] R.C. Brower, H. Neff and K. Orginos, Nucl. Phys. Proc. Suppl. 140 (2005) 686 (hep-lat/0409118) and arXiv:1206:5214.

[6] T. Blum, T. Izubuchi and E. Shintani, arXiv:1208:4349.

[7] H. Yin, PoS (Lattice 2012) 191.

[8] N.H. Christ, et. al., Phys. Rev. Lett. 105 (2010) 241601.

[9] A. Stathopoulos and K. Orginos, SIAM J. Sci. Comput. 32 (2010) 439 , arXiv:0707.0131.

[10] Q. Liu, Columbia University PhD. Thesis, May 2012.

[11] M. Luscher, JHEP 0707 (2007) 081, arXiv:0706.2298.

[12] J. Frison, et. al., RBC-UKQCD Collboration, PoS (Lattice 2013) 460.

[13] A. Juttner, et. al., RBC-UKQCD Collboration, PoS (Lattice 2013) 404.

[14] T. Janowski, et. al., RBC-UKQCD Collboration, Pos Lattice 2013) 402. 